Editor's Note: These short reviews of a recent paper in the Journal, written exclusively by graduate students or postdoctoral fellows, are intended to mimic the journal clubs that exist in your own departments or institutions. For more information on the format and purpose of the Journal Club, please see http://www.jneurosci.org/misc/ifa_features.shtml.

\title{
Habitual Responding and the Dorsal Striatum
}

\author{
Craig A. Schiltz \\ Department of Psychiatry, University of Wisconsin-Madison, Madison, Wisconsin 53719 \\ Review of Vanderschuren et al. (http://www.jneurosci.org/cgi/content/full/25/38/8665)
}

Those of us who were trained to drive with a manual transmission will sometimes step on a nonexistent clutch when braking in a car with an automatic transmission. Although this response is adaptive in the car with manual transmission, it is clearly not useful with an automatic transmission. This everyday example of habitual behavior highlights key elements of automatic or habitual responding. Namely, it is long-lasting and context dependent. The dorsal striatum has been implicated in the consolidation of and execution of such stimulus-response learning. Automatic responses are thought to partially underlie relapses among drug addicts.

In a recent paper in The Journal of Neuroscience, Vanderschuren et al. (2005) explored the role of the dorsal striatum in cue-elicited drug-seeking behavior in rats. Rats were extensively trained to selfadminister intravenous cocaine by lever pressing under a second-order schedule of reinforcement. Second-order schedules of reinforcement engender a high degree of responding for a conditioned stimulus, in this case a light that was contingently paired with responding and delivery of cocaine during training (Fig. 1). On the test day, before the first delivery of cocaine, rats will lever press in the anticipation of cocaine delivery, and this responding is increased with the contingent presentation of the conditioned stimulus.

Received 0ct. 17, 2005; accepted Jan. 5, 2006.

Correspondence should be addressed to Craig A. Schiltz, Department of Psychiatry, University of Wisconsin-Madison, 6001 Research Park Boulevard, Madison, WI 53719. E-mail: cschiltz@wisc.edu.

DOl:10.1523/JNEUROSCI.4419-05.2006

Copyright $\odot$ 2006 Society for Neuroscience $\quad$ 0270-6474/06/261891-02\$15.00/0
This schedule of reinforcement allows the analysis of the effect of cues on drugseeking behavior without the confounding factors caused by the presence of drug in the subject. Additionally, this method avoids extinction of stimulus-response and response-outcome memories that result from nonreinforced lever presses and noncontingent presentation of conditioned stimuli. After rats reached stable performance on the self-administration task they were implanted with indwelling cannulas targeting the dorsal striatum [Vanderschuren et al. (2005), their Fig. 1 (http://www.jneurosci.org/cgi/content/ full/25/38/8665/FIG1)].

Infusion of the dopamine receptor antagonist $\alpha$-flupenthixol into the dorsal striatum dose-dependently decreased cue-elicited responding on the lever that had previously been associated with cocaine infusions. However, there was no effect on responding for inactive lever presses [Vanderschuren et al. (2005), their Fig. 2 (http://www.jneurosci.org/cgi/ content/full/25/38/8665/FIG2)]. Additionally, dorsal striatal infusion of $\alpha$-flupenthixol specifically increased lever pressing for intravenous infusions of cocaine. Given these results, dopamine in the dorsal striatum seems to partially mediate cue-elicited cocaine-seeking behavior and the primary reinforcing effects of cocaine. Infusion of the AMPA/KA receptor antagonist 3SR,4aRS,6RS,8aRS-6-[2(iH-tetrazol-5-yl)ethyl]-1,2,3,4,4a,5,6,7, 8,8a-decahydroiso-quinoline-3-carboxylic acid (LY293558), but not the NMDA receptor antagonist AP-5, also dosedependently decreased cue-elicited lever presses specifically on the lever that was previously associated with cocaine administration. There was no effect of LY293558 for inactive lever presses [Vanderschuren et al. (2005), their Fig. 3 (http://www.jneurosci.org/cgi/content/ full/25/38/8665/FIG3)]. These results argue for a role of AMPA/KA receptor activation in the dorsal striatum in cueelicited cocaine seeking.

The striatum can be divided into limbic (ventral), associative (central), and sensorimotor (dorsal) subcompartments based on their cortical input (Haber et al., 2000). The outputs of the striatum target motor and cognitive action systems and premotor brainstem areas. Hence, the striatum is positioned to play a role in organizing cognitive and motor repertoires to streamline execution of goal-directed tasks on moment-by-moment and longterm bases. For example, electrophysiological recordings in awake, behaving rats learning a procedural T-maze task have shown that striatal neurons undergo a drastic reshaping of their responses during acquisition and overtraining (Jog et al., 1999). Task-related unit responses increase overall during training. In addition, the ensemble response change from one in which task-related activity is distributed throughout the task during learning to one in which task-related activity is exhibited at the start of the task and after goal attainment after learning.

The dorsal striatum is also progressively recruited during learning of motor skills. Humans trained to execute a sequenced finger-tapping task exhibit increasing dorsal striatal activity and 
decreasing ventral striatal activity (Lehericy et al., 2005). A similar recruitment of activity within dorsal striatal, or sensorimotor, subregions is seen in monkeys chronically self-administering cocaine compared with monkeys that are relatively new to the task (Porrino et al., 2004). Successive engagement of more dorsal subregions may occur via glutamate-mediated plasticity modulated by dopamine. The involvement of dopamine is suggested by the ventromedial to dorsolateral ascending loops reciprocally connecting the striatum with its dopaminergic innervation in the ventral tegmental area and the substantia nigra (Haber et al., 2000). This anatomical organization may also explain why contingent presentation of the conditioned stimulus elicits dorsal striatal dopamine efflux, whereas noncontingent presentation of the conditioned stimulus elicits ventral striatal dopamine efflux after extensive training. Regardless of the mechanism, the shift in task-related activity may be adaptive in that performance of well learned tasks no longer necessitates access to frontal cortical striatal systems mediating attentional processes, thereby freeing these resources for use in unlearned or more immediately pressing tasks (Lehericy et al., 2005).

It is important to use models such as the one used by Vanderschuren et al. (2005) because they have real world validity: habitual responses are part of addiction. For example, the development and execution of habit learning can happen in the absence of declarative memory (Bayley et al., 2005). These observations may explain why drug abusers do not recog-
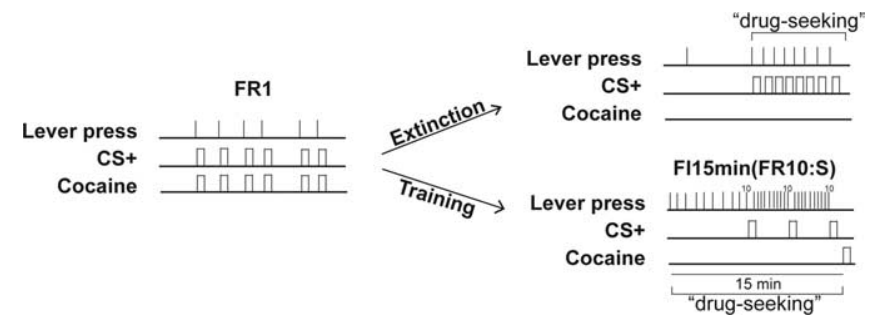

Figure 1. Schematic diagram of different schedules used to study cue-elicited drug-seeking behavior. First, rats are trained on a fixed-ratio (FR1) schedule in which every lever press is rewarded with an infusion of cocaine paired with the presentation of a conditioned stimulus (CS; a light). The top arrow illustrates one method of studying cue-elicited drug-seeking behavior. After several extinction trials during which lever presses are not reinforced with cocaine infusions or presentation of the CS, lever pressing decreases. If the $C S$ is then contingently presented, rates of lever pressing increase demonstrating cue-elicited drugseeking behavior. The bottom arrow illustrates a second-order schedule of reinforcement. After preliminary training, rats are trained to lever press during a fixed interval ( $\mathrm{Fl} ; 15 \mathrm{~min}$ in this study), during which time they do not receive cocaine. If the rat responds 10 times, the $C S$ is presented. Because CS presentation invigorates responding, this lever pressing is termed cue-elicited drug-seeking behavior. The rats have been trained so that completion of 10 responses and delivery of the CS are necessary for the delivery of cocaine at the end of the fixed interval. This method avoids confounds of extinction training and noncontingent $C S$ presentation, which may alter stimulus-response and response- outcome associations.

nize the transition into addiction and why addicts describe drug-seeking behavior as being on "auto-pilot." Although Vanderschuren et al. (2005) did not assess the degree to which their rats were insensitive to reinforcer devaluation, an operational definition of habitual responding, the lengthy training period needed and high degree of responding generated by second-order schedules of reinforcement almost guarantee robust habit learning. Through their model, the authors demonstrated the contribution of dorsal striatal dopamine transmission in cocaine reinforcement as well as that of dopamine and glutamate transmission in cue-elicited cocaine-seeking behavior.

\section{References}

Bayley PJ, Frascino JC, Squire LR (2005) Robust habit learning in the absence of awareness and independent of the medial temporal lobe. Nature 436:550-553.

Haber SN, Fudge JL, McFarland NR (2000) Striatonigrostriatal pathways in primates form an ascending spiral from the shell to the dorsolateral striatum. J Neurosci 20:2369-2382.

Jog MS, Kubota Y, Connolly CI, Hillegaart V, Graybiel AM (1999) Building neural representations of habits. Science 286:1745-1749.

Lehericy S, Benali H, Van de Moortele PF, Pelegrini-Issac M, Waechter T, Ugurbil K, Doyon J (2005) Distinct basal ganglia territories are engaged in early and advanced motor sequence learning. Proc Natl Acad Sci USA 102:12566-12571.

Porrino LJ, Lyons D, Smith HR, Daunais JB, Nader MA (2004) Cocaine self-administration produces a progressive involvement of limbic, association, and sensorimotor striatal domains. J Neurosci 24:3554-3562.

Vanderschuren LJ, Di Ciano P, Everitt BJ (2005) Involvement of the dorsal striatum in cuecontrolled cocaine seeking. J Neurosci 25:8665-8670. 